\title{
Lack of Evidence Does Not Equal Lack of Benefit: Neoadjuvant Chemotherapy and Trimodality Therapy in Selected Patients with Muscle-Invasive Bladder Cancer
}

\author{
In response to: Dirk Böhmer and Arne Grün. Lacking Evidence to Recommend Neoadjuvant \\ Chemotherapy and Definitive Radiotherapy in Muscle-Invasive Bladder Cancer
}

\author{
Di Maria Jiang ${ }^{1} \cdot$ Peter Chung ${ }^{2} \cdot$ Girish S. Kulkarni $^{3} \cdot$ Nick D. James $^{4,5} \cdot$ Srikala S. Sridhar $^{1}$ \\ Published online: 3 March 2021 \\ (C) The Author(s), under exclusive licence to Springer Science+Business Media, LLC part of Springer Nature 2021
}

\section{Dear Editor,}

Thank you for the opportunity to address the role of neoadjuvant chemotherapy (NAC) prior to bladder-sparing trimodality therapy (TMT) for patients with muscle invasive bladder cancer (MIBC), in response to the letter by colleagues Böhmer and Grün. While we agree the role of NAC in this setting is not clearly defined, there are several important reasons for considering this treatment approach in select patients who are planned for TMT.

Historically, TMT was reserved for the elderly and frail patients with MIBC who were ineligible for radical cystectomy (RC). These patients were often also ineligible for cisplatinbased NAC due to significant comorbidities or poor performance status. Thus, NAC was rarely used in historical TMT trials. In the contemporary setting however, TMT is being increasingly utilized as an accepted alternative to RC and in selected patients has shown comparable long-term outcomes. There are now more

Srikala S. Sridhar

srikala.sridhar@uhn.ca

1 Department of Medicine, Division of Medical Oncology, Princess Margaret Cancer Center, University Health Network, University of Toronto, 700 University Avenue, Toronto, Ontario M5G 6M9, Canada

2 Radiation Medicine Program, Princess Margaret Cancer Centre, Department of Radiation Oncology, University of Toronto, Toronto, Canada

3 Division of Urology, Departments of Surgery and Surgical Oncology, Princess Margaret Cancer Centre, University Health Network, University of Toronto, Toronto, Ontario, Canada

4 Radiotherapy and Imaging Division, The Institute of Cancer Research, London, UK

5 University Hospitals Birmingham NHS foundation Trust, Birmingham, UK patients who are younger and fitter opting for TMT. [1]. Importantly these patients, unlike the older and frailer patients, are more likely to be eligible for and tolerate NAC.

There is strong biological rationale and Level 1 evidence supporting the use of NAC in MIBC. This is a highly chemosensitive disease with a high propensity for distant relapse, likely due to the presence of micrometastatic disease at presentation. The goal of NAC prior either RC or TMT is essentially similar - to eliminate micrometastatic disease and improve cure rates. Cisplatin-based NAC has a pathologic complete response (pCR) rate of about $30 \%$ which is strongly correlated with improved overall survival (OS) [2].

The question remains, why would NAC improve OS in patients treated with RC but not with TMT? According to the landmark $\mathrm{ABC}$ meta-analysis, $\mathrm{NAC}$ has been shown to improve OS, regardless of whether patients receive RC or radiation [3]. In the large BC2001 phase III clinical trial evaluating the added benefit of concurrent 5-fluorouracil plus mitomycin to radiotherapy, the use of NAC did not impact the benefit of concurrent chemotherapy [4]. While concurrent chemotherapy improves local control, it did not improve metastasis-free survival or OS. This suggests that concurrent chemotherapy cannot serve as a substitute for NAC and does not adequately address the risk of micrometastases at presentation.

The BA06 30894 trial was a randomized phase III trial which evaluated the benefit of cisplatin, methotrexate, and vinblastine (CMV) NAC for 3 cycles prior to local definitive therapy and accrued patients between 1989 and 1995. The results showed that NAC reduced the risk of death by $26 \%$ for patients who received RC and by $20 \%$ for patients who received radiotherapy alone [5]. As Böhmer and Grün have pointed out, statistical significance was seen in patients undergoing RC but was not seen among patients who received radiotherapy; nevertheless, a strong trend in support of NAC 
was observed $(p=0.070)$. There are also a number of important caveats that warrant consideration. The BA06 30894 trial was not designed to specifically evaluate the benefit of NAC in either subgroup, but rather was powered to detect a $10 \%$ benefit at 2 years across the whole study population. This degree of benefit was not realized, as the initial report demonstrated only a 5.5\% OS improvement at 3 years. The updated analysis after a median follow up of 8 years, however confirmed an overall survival advantage [8]. To correctly interpret whether the overall treatment effect is heterogenous across subgroups, an interaction analysis should be used rather than the $p$ value [6]. Based on the results of their pre-planned (exploratory) interaction analysis, "for overall survival, there was no evidence to suggest that the effect of chemotherapy was more or less for patients treated with cystectomy than for those treated with radiotherapy" $[7,8]$. As Böhmer and Grün acknowledged, baseline characteristics were not well balanced between the RC and radiotherapy groups. This was expected since the choice of local definitive therapy was not randomly assigned. Patients in the radiotherapy arm were older, frailer, and had more advanced disease, and were a different patient population than patients who received RC. However in patients who were eligible to receive NAC, the benefit of NAC is supported by the results of the interaction analysis [8]. With respect to locoregional disease-free survival, the $9 \%$ improvement in the radiotherapy subgroup compared with $26 \%$ in the $\mathrm{RC}$ subgroup is hardly a surprising finding given that radiotherapy alone and not concurrent chemoradiotherapy was used in this trial. Concurrent chemoradiotherapy is now recognized as the standard of care for trimodality therapy and has shown superior local control over radiotherapy alone based on level I evidence [4].

In a Danish trial, the addition of 3 cycles of cisplatin-based NAC to radiotherapy alone in 120 patients improved median survival from 16.3 to 19.2 months, although statistical significance was not reached likely due to inadequate sample size [9].

There is not yet any robust data evaluating the benefit of NAC prior to radiotherapy concurrent with chemotherapy. As discussed in our review, the Radiation Therapy Oncology Group (RTOG) 89-03 phase III trial published in 1998 which randomized patients to two cycles of neoadjuvant CMV followed by TMT versus TMT alone was significantly underpowered, with only 123 patients randomized. Thus, RTOG 89-03 cannot exclude the benefit of NAC prior to TMT given its limited statistical power. At the time of trial conduct, growth factor support and modern antiemetics were not yet available, and completion rates for NAC were only $67 \%$. We recently published our center's experience in using NAC prior to TMT. Although many patients required dose adjustments, completion rates of NAC were $95 \%$. All patients completed planned radiotherapy, and $84 \%$ completed at least $60 \%$ of planned concurrent chemotherapy [10]. More modern trials evaluating NAC also report favorable toxicity profiles, with grade $3-4$ toxicity of NAC between 10 and $15 \%[11,12]$. In addition, RTOG 89-03 did not provide any evidence as to whether three or four (instead of two) cycles of NAC can improve cure rates. Therefore, no definitive conclusions can be drawn from this trial.

Although, currently, no large randomized phase III trial has been able to address this important question of whether there is a benefit to NAC prior to TMT, the "lack" of evidence does not necessarily imply lack of benefit. NAC continues to be grossly underutilized in the treatment of MIBC and outcomes remain suboptimal [13-15]. Administering NAC prior to TMT in selected patients is a safe and feasible strategy which has the potential to improve survival. Ongoing trials are already increasingly incorporating the use of NAC prior to TMT, as we highlighted. In particular, the RETAIN bladder phase II trial (NCT02710734) is evaluating a risk adapted approach to help guide various bladder sparing strategies based on response to NAC and tumor mutational profile. The results of these trials are eagerly awaited to further define the role of NAC prior to TMT in the genomic era.

\section{Declarations}

Conflict of Interest DMJ has no conflict of interest to declare. PC has received honoraria from AbbVie, AstraZeneca, and research funding from Sanofi. GSK has participated in advisory boards for Roche, Merck, Janssen, Theralase, and Ferring; received honoraria from TerSera, Abbvie, and Sanofi; and received funding for clinical trial activities from Astra Zeneca, Merck, BMS, Theralase, and Janssen. NDJ has received consultancy fees from AZ, Merck, and Roche plus trial support from AZ for my RADIO trial. SSS has received compensation from Roche, Merck, Pfizer, Bayer, BMS, Astellas, and AstraZeneca for service as a consultant and has received research funding from Janssen and Sanofi.

\section{References}

1. Wilhite TJ, Routman D, Arnett AL, et al. Increased utilization of external beam radiotherapy relative to cystectomy for localized, muscle-invasive bladder cancer: a SEER analysis. Bladder. 2018;5(3):34. https://doi.org/10.14440/bladder.2018.639.

2. Petrelli F, Coinu A, Cabiddu M, Ghilardi M, Vavassori I, Barni S. Correlation of pathologic complete response with survival after neoadjuvant chemotherapy in bladder cancer treated with cystectomy: a meta-analysis. Eur Urol. 2014;65(2):350-7. https:// doi.org/10.1016/j.eururo.2013.06.049.

3. Advanced Bladder Cancer (ABC) Meta-Analysis Collaborators. Neoadjuvant chemotherapy in invasive bladder cancer : update of a systematic review and meta-analysis of individual patient data. Eur Urol. 2005;48:202-6. https://doi.org/10.1016/j.eururo.2005.04.006.

4. James N, Hussain S, Hall E. Radiotherapy with or without chemotherapy in muscle-invasive bladder cancer. N Engl J Med. 2012;63: 1477-88. https://doi.org/10.1016/j.eururo.2012.12.008.

5. International collaboration of trialists. International phase III trial assessing neoadjuvant cisplatin, methotrexate, and vinblastine chemotherapy for muscle-invasive bladder cancer: Long-term results of the BA06 30894 trial. J Clin Oncol. 2011;29(16):2171-7. https:// doi.org/10.1200/JCO.2010.32.3139. 
6. Spears MR, James ND, Sydes MR. 'Thursday's child has far to go'interpreting subgroups and the STAMPEDE trial. Ann Oncol. 2017;28(10):2327-30. https://doi.org/10.1093/annonc/mdx410.

7. International collaboration of trialists. Neoadjuvant cisplatin, methotrexate, and vinblastine chemotherapy for muscle-invasive bladder cancer: a randomised controlled trial. Lancet. 1999;354(9178):53340. https://doi.org/10.1016/S0140-6736(99)02292-8.

8. Griffiths G. International phase III trial assessing neoadjuvant cisplatin, methotrexate, and vinblastine chemotherapy for muscleinvasive bladder cancer: long-term results of the BA06 30894 trial. J Clin Oncol. 2011;29(16):2171-7. https://doi.org/10.1200/JCO. 2010.32.3139.

9. Sengeløv L, Von Der Maase H, Lundbeck F, et al. Neoadjuvant chemotherapy with cisplatin and methotrexate in patients with muscle-invasive bladder tumours. Acta Oncol (Madr). $2009 ; 41(5): 447-56$. https://doi.org/10.1080/ 028418602320405041 .

10. Jiang DM, Jiang H, Chung PWM, et al. Neoadjuvant chemotherapy before bladder-sparing chemoradiotherapy in patients with nonmetastatic muscle-invasive bladder cancer. Clin Genitourin Cancer. 2019;17(1):38-45. https://doi.org/10.1016/j.clgc.2018.09.021.

11. Plimack ER, Hoffman-Censits JH, Viterbo R, Trabulsi EJ, Ross EA, Greenberg RE, et al. Accelerated methotrexate, vinblastine, doxorubicin, and cisplatin is safe, effective, and efficient neoadjuvant treatment for muscle-invasive bladder cancer: results of a multicenter phase II study with molecular correlates of response and toxicity. J Clin Oncol. 2014;32(18):1895-901. https://doi.org/ 10.1200/JCO.2013.53.2465.

12. Choueiri TK, Jacobus S, Bellmunt J, Qu A, Appleman LJ, Tretter $\mathrm{C}$, et al. Neoadjuvant dose-dense methotrexate, vinblastine, doxorubicin, and cisplatin with pegfilgrastim support in muscle-invasive urothelial cancer: Pathologic, radiologic, and biomarker correlates. J Clin Oncol. 2014;32(18):1889-94. https://doi.org/10.1200/JCO. 2013.52.4785.

13. Fedeli U, Fedewa SA, Ward EM. Treatment of muscle invasive bladder cancer: evidence from the National Cancer Database, 2003 to 2007. J Urol. 2011;185(1):72-8. https://doi.org/10.1016/j. juro.2010.09.015.

14. Booth CM, Siemens DR, Peng Y, Tannock IF, Mackillop WJ. Delivery of perioperative chemotherapy for bladder cancer in routine clinical practice. Ann Oncol. 2014;25(9):1783-8. https://doi. org/10.1093/annonc/mdu204.

15. Huo J, Ray-zack MD, Shan Y, et al. Discerning patterns and quality of neoadjuvant chemotherapy use among patients with muscleinvasive bladder cancer. Eur Urol Oncol. 2018. https://doi.org/10. 1016/j.euo.2018.07.009.

Publisher's Note Springer Nature remains neutral with regard to jurisdictional claims in published maps and institutional affiliations. 